\title{
The Organizational Relational model: proposal and results ${ }^{*}$
}

\author{
P. Migliarese \\ Università della Calabria e Politecnico di Milano \\ P.zza Leonardo da Vinci, 32 - 20133 Milano - Italy \\ Fax +39.2.2399.2720 \\ e-mail migliare@mail.ecopro.polimi.it \\ C. Ferioli \\ Politecnico di Milano \\ P.zza Leonardo da Vinci, 32 - 20133 Milano - Italy \\ Fax +39.2.2399.2720 \\ e-mailferioli@mail.ecopro.polimi.it
}

\begin{abstract}
The paper proposes a new method, the Organizational Relational model, based on the new concept of Relation, for the analysis of innovative organizational models like the network organization. The proposal is supported through results and through the evidence from some applications in real situations. Two different basic elements can be distinguished in the innovative organization: nodes (i.e. organizational units or agents) and links among nodes (i.e. organizational relations, contracts, communication channels or architectures). This paper explains the important role of IT in providing tools for the innovative organizations. IT and especially GDSS extend the autonomy of nodes and provide instruments for supporting the links between two (or more) nodes. The proposed method describes the relation according to four elements: the tools supporting the link, the goals of the node, the organizational rules and norms of the organizational group and the organizational culture. Moreover, the proposed method provides a "guide" based on the oppositions formal versus informal and society versus community for describing the role of IT (especially GDSS) in innovative organizational models. The method has been applied in different organizational contexts, like public health and various industrial companies. While the specific applications are described and detailed in other papers, the empirical evidences and the synthetic results obtained
\end{abstract}

\footnotetext{
- The paper is due to the collaboration of the authors. Nevertheless, par. 1, 4 and 5 are common, par. 2 is mainly due to Piero Migliarese, par. 3 is mainly due to Claudio Ferioli.
} 
are described in this paper as a confirmation of the proposed organizational framework and method.

\section{Keywords}

Innovative organizational models, network organizational models, relational organizational model, organisational networks, job modification, organisational culture

\section{INTRODUCTION}

In the research on new models of organization, network enterprise or network organizational models are emerging and largely successful concepts. Two different types can be distinguished with respect to the network organization: external networks, in which several distinct companies are linked in various forms and internal networks, in which distinct organizational units of the same company have particular managerial autonomy and are linked by complex relations apart from the hierarchical one.

In the last few years research on dynamic networks has grown rapidly and valuable results have been achieved in industrial organization theory with regard to the forms of networks in different enterprises and the causes that lead to the growth and development of inter-firm networks (Miles and Snow, 1986). External networks have been described and analysed in detail, and many examples and theories have been provided in the literature. However, internal networks research has given fewer results and few studies have been provided.

Nevertheless, many recent signals from companies suggest that a model of network organization is really emerging in the industrial world. Many companies have brought changes and innovation in their organizations: we think that in many cases the model of internal network organization can describe the resulting organizational forms.

Some of these changes involve the use of self-managed teams such as, in the manufacturing area, the U.T.E.s (Technological Elementary Units) of F.I.A.T., the use of the internal market mechanism to regulate interchanges among different organizational units in the same company, the disappearance of clear firm borders with the growth of units located neither inside nor outside the factory, such as some suppliers of FIAT Auto, which are neither inside the organization, since they are legally autonomous, nor completely outside, since they physically work in the area of the customer (Cesaria, 1993).

It is possible to say that actual trends in organizations are towards informationbased as well as knowledge-based organizations, and that organizational units are largely composed of specialists. Employees are encouraged to act in an entrepreneurial way, as they have to achieve the objective assigned to their organizational unit; in particular they can obtain the know-how and the ability required for the assigned objectives through relations with colleagues and through 
interchange of services or information with other organizational units (Drucker, 1988).

In the present competitive environment, innovative organizational forms seem to enlarge the autonomy of nodes and seem to foster the establishment of complex links among nodes for improving know-how, flexibility and capacity to react rapidly to environmental changes.

The internal network organization is considered here with reference to two basic elements: the autonomy of nodes and the link complexity.

Nodes can be defined both as organizational units and as agents. The need for enlarged autonomy of nodes is one of the most relevant aspects of the new organizational forms which have emerged in the last few years. The need for autonomy is due to the new competitive features in the present economic environment. The enlarged autonomy requires improvement of the skills and the know-how of every node. This paper explains the role of IT in supporting autonomy of nodes. In particular, DSS and GDSS provide methodology for problem solving, tools for decision making and instruments for sharing knowledge: in this way they increase the capacity and the know-how of the nodes and they allow the enlargement of the autonomy of nodes.

The second aspect of network organization regards the capability of nodes in creating complex links. Every node can obtain the capacity and the know-how required for its objectives through making links with other nodes: the link allows the exchange of information, services, know-how, etc., between two (or more) nodes through complex organizational relations or communication architectures and channels or special contracts. The paper explains the role of IT and GDSS in supporting the links in network organizations: IT establishes a channel of communication between two (or more) nodes and provides facilities for the exchange of information. For this reason, IT as well as GDSS is one of the most powerful instruments for creating links among nodes.

Moreover the paper describes a specific method for the analysis and the design of network organizations based on the concept of relation (a relational approach to the network organization). A relation is the result of the connection of two nodes: schematically, the relation is made up of the two nodes together with the links that connect the two nodes. The relation considers both properties of the nodes and characteristics of the link itself. The paper proposes a method for the analysis of relations derived from the AGIL model (Adaptation, Goal, Integration, Latency). This model, proposed by T. Parsons for sociological analysis, has been modified for the analysis of the relation in the network organization at micro-analysis level.

In the proposed modified AGIL method, the relation is described according to four components: the tools that support the connection, and that enable organizational units. to react to the environmental variables (Adaptation to the environment); the goals of the nodes for which the relation is created; the organizational rules and norms that allow the Integration of individuals in organizational groups, and the Latent organizational culture. 
Concerning the design of IT systems within a network organization, the paper proposes two basic dilemmas: formal/informal and community/society. The dilemma formal/informal considers the presence in each of the four components of formal elements and of informal ones. The opposition community/society was introduced by F. Tonnies for sociological analysis. The word community indicates aggregations based on traditional behaviour and common will of people, while society considered to be based on common accords, on legal rules and on contracts. The paper proposes to examine the role of GDSS and IT in designing the relations in network organizations: along the two basic dilemmas. Information Technology amplifies formal characters and societal aspects, and reduces informal and community ones. In this way IT improves the efficiency of the relation, but reduces the richness of the interaction: the design of IT tools has to solve the trade-off between these two aspects.

The paper is organized as follows: section two describes the two basic elements of the network organization: the nodes and the links among nodes. A method (the relational method) is proposed for the analysis and the design of network organization, based on the concept of relation. The concept of relation refers both to the link and to the nodes connected by the link. First of all, the proposed method provides the scheme of the four basic elements of the relation: the tools, the goals, the organizational rules, the organizational culture. Secondly, the proposed method suggests a guide for the analysis of the four basic elements of the relation, based on the opposition formalinformal and on the opposition community/society.

In section three, the paper explains the role of IT in the network organization. IT (especially DSS and GDSS) provides support both to autonomy of nodes and to the establishment of links. As regards autonomy of nodes, IT (especially DSS and GDSS) improves the know-how, the problem solving ability and the decision making capacity of nodes. The paper describes the example of Delta methodology, a technique implemented in Digital and based on distributed data bases connected with the computer network. As regards links, IT and especially GDSS provide different tools that support the creation of links between two (or more) nodes and facilitate the communication through the links.

In section four, applications of the proposed relational model are briefly outlined. In particular a successful IT application, used in the health service sector is presented as well as some applications in manufacturing enterprise environments. The use of the relational method was helpful in the design process of an IT system for analyzing the modification of the "organizational relations" among the main actors in the health service organization. Other successful applications concern empirical research conducted in a sample of enterprises (AT\&T, Olivetti, Digital) for analyzing of an existence and effectiveness of network organizations. This empirical research revealed that the presence of network organization was positively correlated with effectiveness described in the section, moreover, a case study conducted in the Italian company Olivetti, in which the existence of several "relations" among organizational units can be discovered and explained with the 
proposed Relational Model. While the concept of network organization is somehow not totally defined, the "relational organizational model" shows a great explanation power and the concept of relation proved very helpful in analyzing traditional as well as innovative organizational forms. Conclusions follow.

\section{THE RELATIONAL MODEL: A NEW MODEL PROPOSED FOR ORGANIZATIONAL ANALYSIS}

New competitive and environmental features require new models for organizational analysis. Traditional models miss some of the new requisite for organizational design. For example, managers have to design flexible organizations, based on dispersed organizational units located in all over the world; Information Technology has became the main system for co-ordination and communication; companies adopt new mixed strategies of collaboration and competition.

Organizational designers need to integrate their competencies with new tools and new perspectives. Traditional organization charts represent only hierarchical lines of control and communication: they are no longer adequate for describing organizations composed of semi-autonomous organizational units, that can create several links with other organizational units (as well as with other companies) through lateral mechanisms of co-ordination and communication.

New client-supplier links provide another example. In several cases the reduction of slack-resources has drastically changed this tie from the classical market exchange to a new mechanism. New client-supplier links are based on several aspects, such as: (i) the definition of some common goals (partial economic-risk sharing, objectives of product quality improvements, etc.), (ii) long period contracts, (iii) strong interpersonal trust, (iv) organizational exchange (knowledge sharing, information exchange, etc.), (v) technical links, such as computer networks, etc. (Lamming, 1993).

Traditionally, client-supplier links have been studied through the analysis of economic exchange based on the concept of economic transaction (Williamson, 1975; Jensen and Meckling, 1976; Alchian and Demsez, 1972). But nowadays the transaction-costs model can provide only a partial description of these new links, because it considers only economic elements and disregards technical, social and organizational aspects.

\subsection{The proposed Relational model for organizational analysis}

We propose a new model for organizational analysis, the relational model of organization. The researches on this new model are still in progress, nevertheless some significant theoretical and practical results have been achieved. The Relational model for organizational analysis describes the organizations as networks of nodes linked through organizational relations. 
Nodes are composed of organizational actors. Several criteria may be defined for the location of nodes: formal borders of organizational units, similarity in technical activity, equality in hierarchical position, similarity in professional qualification, etc. The choice of the best criteria may vary according to the purpose of the analysis.

For example, for the design of a Decision Support System supporting technical activities, organizational actors will be grouped in nodes according to their technical qualifications. Instead, if the purpose of the analysis is the implementation of an IT support for lateral communication, the best criteria might be the relational homogeneity of nodes, i.e., defining the nodes so that all the components of a node have the same set of relations with every other organizational actor (Baker, 1992).

Moreover the width of the nodes varies in relation to the size of the analysis (Ferioli and Migliarese, 1994). For example, if the analysis covers a whole organization with many divisions located in several countries, a local factory may be considered a node; whereas if the analysis is limited to a shop floor, the size of nodes is restricted to the individual employee.

Each node is linked with other nodes through a complex set of connections (see for example the previously described client-supplier link), that is described as organizational relation.

The word "relation" was firstly introduced in organization theory by Elton Mayo and his Human Relations school. Even if nobody can deny the importance of this school in organization studies, the concept of relation is used here with a limited meaning: (1) only interpersonal relations are considered; (2) the analysis is centred on the organizational climate, whereas the relation remains only in the background. Some other authors (Gabarro, 1990; Granovetter, 1992; Ferioli and Migliarese, 1994) have proposed instead the concept of organizational relation as the basis for organizational analysis.

The organizational relation is defined as a link between two organizational actors (individuals and/or organizational units and/or different companies) with the following characteristics: (a) it is orientated toward a (partially) common goal, (b) it is not trivial, (c) it is not an occasional link, (d) it is supported by several tools.

(a) Two economic actors establish an organizational relation for pursuing a (partially) shared goal. For example, the client-supplier link becomes an organizational relation if client and supplier identify a common objective (such as a product quality improvement; a reciprocal economic aid; etc.). If the shared goal disappears, the organizational relation dies or changes to another mechanism (for example to a classical market contract).

(b) The shared goals of the organizational relation are jointed to the objectives of the organization (for example productivity objectives, market objectives, etc.). This means that the organizational relation is not "trivial", but created by the nodes in 
order to obtain their organizational objectives. This not "trivial" feature distinguishes organizational relations from pure phenomenological, interpersonal interactions, that (roughly speaking) are not linked to any organizational objectives.

(c) A link requires a medium/long period to become an organizational relation.

(d) The organizational relation is supported by several tools, such as: formal contracts, interpersonal links, exchange of materials/services, exchange of information, etc.

Table 1 The definition of relation

- It is based on a (partially) common goal

- It is not trivial

- It is not an occasional link

- It is supported by several tools

\subsection{The design method of organizational relations}

A method for the design of organisational relation has been proposed by the authors. This method describes organizational relations through four axes: the tools supporting the relation, the goal(s) common to the economic actors, the norms regulating the behaviour of actors within the relation and the cultural background associated to the relation. They represent the four base elements common to every organizational relation, whereas they do not provide the real description of organizational relations (For example a client-supplier relation has a different "shape" from a relation between two single employees. Nevertheless the four axes position the analysis and the design for both relations)

\section{Tools}

The axis of tools regards the necessary instruments for a relation to take place $\alpha_{i}$ : the support to improve relation effectiveness. Different kinds of tools are used in organizations for supporting collaborative relations or for enabling the creation of relations:

Inter-personal contacts are the main instruments for many collaborative relations, so that many personnel executives try to promote this type of interaction. Some of the instruments for fostering interpersonal contact are (Hastings, 1993):

- periodic meetings for people from different divisions;

- residential training periods for new employees (6-9 months);

- personnel rotation (also for limited period); etc. 
Group management techniques and working group methodologies are tools that improve group work collaboration, and they are required for every group leader.

IT instruments are tools for supporting and creating communication channels among economic/organizational actors.

The price system in the internal market mechanism provides instruments for establishing relations among organizational units, so it can be classified as a tool.

According to Miles and Snow (1992), varicus companies are using an internal market mechanism for regulating internal interactions: some organizational units play the role of brokers and buy services/goods from other organizational units. Through this new rule system, the brokers create some particular product required by clients.

\section{Goals}

The axis of goals explains the purposes and the objectives for which an organizational relation is established and maintained. The presence of a shared goal is the raison d'être of the organizational relation: no effective relation can survive without defined goals. The previously described client-supplier relations are different from classical client-supplier market contracts because they are based on (partially) common objectives (common design of new products, shared objectives of quality improvement, joint investments in new plants, etc.). If the goal disappeared, the relation would change into another type of link (for example in a simple market exchange).

Moreover, the relation goals influence the remaining characteristics of the relation: they determine the amount of information to exchange, the need for negotiating mechanism and the required cultural background common to the actors involved in the relation (if the goals of the two persons/organizational units are different, the relation will require a negotiation and the creation of complex contractual norms and rules).

\section{Rules}

The axis of rules and norms defines the set of implicit as well as explicit rules that govern relations. Relational norms define the accepted behaviour: in this way they allow/prevent the integration of (new) actors in the group created through the relation. Relations are regulated either by general organizational rules and values, and by specific norms defined among involved organizational actors.

For example, the organizational relations based on internal market mechanisms are regulated through: (i) specific contracts -negotiated by the organizational units that create the relation, and (ii) general rules, that the headquarters has decided (for example) in order to promoting some company areas.

In most cases, many organizational rules are "implicit" and based on unwritten social values. In some organizations hierarchic relations are very formal and "cold", while in others they take place in an informal and friendly way. 


\section{Cultural background}

The cultural background is composed of the organizational culture common to the two economic/organizational actors involved in the relation, i.e., the values, the traditions and the customs the two actors share.

The cultural background forms the base knowledge shared by the actors of the relation, i.e., the common assumptions that do not require any negotiation or contractual definition (Schein, 1984). A largely shared cultural background improves the effectiveness of organizational relations, as it reduces the need for negotiation and information exchange.

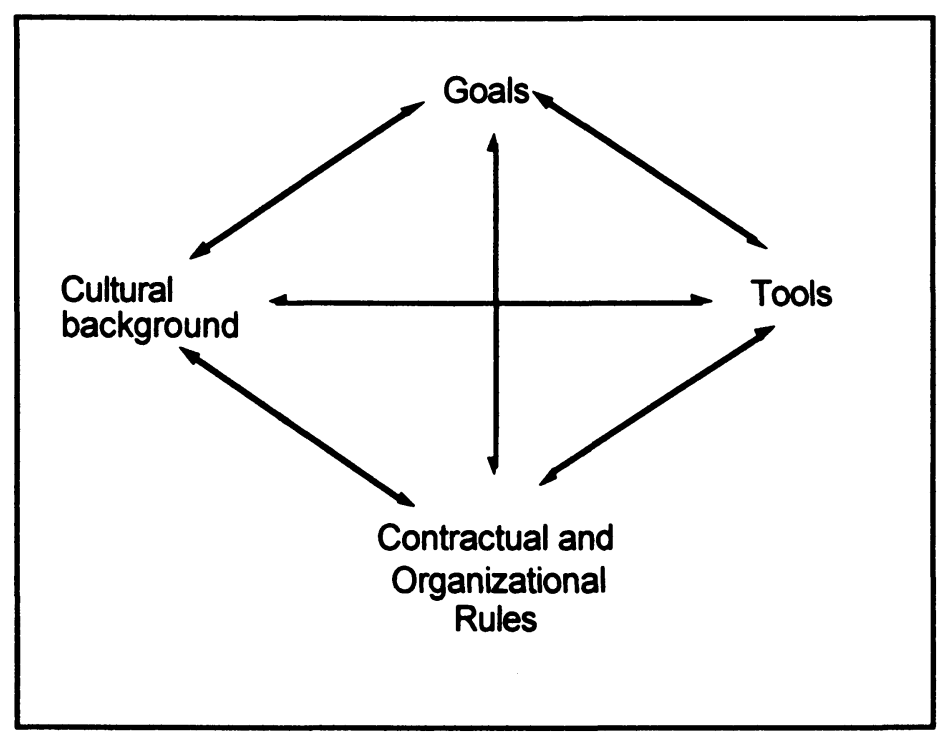

Figure 1 The four axes of the organizational relation according to the proposed method (Ferioli and Migliarese, 1994)

\section{THE NEW ROLE OF IT AS RELATIONAL TECHNOLOGIES}

The proposed Relational Perspective for the analysis of organizations provides indications for the design of IT supports and for their introduction in organizations. Information Technology plays the new role of relational technologies in new organizational models because it facilitates the creation of a network of organizational relations. 


\subsection{IT support for creation of nodes}

IT based tools foster the constitution of semi-autonomous nodes, through providing support to technical and managerial decisions (DSS, MIS, EIS, etc.) that improve the decisional capabilities of managers as well as the competencies of organizational units.

Decisional support for management_should facilitate managers to play the new role of small businessman, assigned to them by new competitive features (Leidner and Elam, 1993). Roughly speaking, these systems should provide two kinds of information:

- Data about organizational units subordinate to the manager in order to facilitate the control over a large number of employees (increased span of control) and the monitoring of the performance of organizational units (through economic and/or technical indicators);

- Data from outside the organizational unit, in order to facilitate the middle manager to act as an "entrepreneur". This requires the capability to respond to new opportunities/ties from the environment (that can be fostered for example with information about the economic environment, the technical/scientific community, etc.) as well as behaviour in line with the strategy and the main decisions of the company (that can be facilitated through data about main company decisions, market/economic company results, etc.).

Decisional supports for organizational units enlarge the capability of autonomous problem solving and decision making. Organizational units require an enlarged amount of information and know-how for solving complex but well-known problems. Decision Support Systems provide partial solutions to this need for knowledge (Ferioli and Migliarese, 1994). Roughly speaking, knowledge based DSS can provide organizational units with the technical capability for complex problem solving, through the "collection" of existing expertise and know-how. For example, a maintenance team whose activity is supported by a DSS has less need for interactions with other maintenance units for problem solving or sharing experience. All the know-how required is collected in the IT support, and the team is completely autonomous.

Moreover, organizational units need to improve their expertise and creativity, for finding valid solutions for new problems. Tools based on Artificial Intelligence can improve the capability of discovering new solutions for emerging problems, that were never faced before. For example, applications based on "neural networks" or on "genetic algorithms" can find hidden dependencies among data, build new models, etc. 


\subsection{IT support for the creation of organizational relations}

IT can facilitate the creation of organizational relations among nodes, mainly through providing support to lateral mechanisms of communication and coordination. These mechanisms are based on the exchange of information among organizational units through channels different from hierarchy-based links. Lateral mechanisms are established by organizational units in order to pursue some specific organizational goal (for example: to buy services, to obtain information/solutions about a technical problem, etc.). The goal influences the "pattern" of the link. For example a production team (liable for a part of the production process, for example door assembling in a car production process) may establish links with:

- (link-1) the engineering office and the quality manager, for suggesting a door modification (this link may be based on a meeting);

- (link-2) the maintenance team, for planning plant servicing (this link may be based on a periodic planned meeting);

- (link-3) a similar production team, located in another plant, for solving a technical problem (this link may be based on interpersonal contact); etc.

Two main problems may affect communication in these links: (a) organizational units may be located in remote places and (b) the identification of "suitable" organizational units sometimes may be difficult (for example in link-3).

The elimination of communication obstacles (first problem) is one of the main areas of IT based application: IT communication tools solve the time and space constraints of communication among organizational units or even among different companies. Research on the field of Group Decision Support Systems (Nunamaker, Vogel and Konsynski, 1989; Gallupe, Bastianutti and Cooper, 1991) and on Computer Supported Cooperative Work (Bannon, 1997) provide important tools in this area.

Information Technology may also provide (partially) solutions for the problem of identifying the "suitable" organizational unit (second problem). In particular, Organizational Decision Support Systems (Lee, McCosh and Migliarese, 1988) collect information about the organizational units and the personnel belonging to the organization, their expertise, the organizational rules and procedures, etc. They provide a "map" of the organization and facilitate the research of the unit/person with particular characteristics.

An example of ODSS, provided by British Petroleum (Hastings, 1993) is outlined in figure 2. The BP high technology environmental department (called HSE technology team) provides knowledge and expertise for solving environmental problems and for preventing disasters. Those kinds of solutions are used by BP itself and are sold to BP's market client .The HSE technology team is composed of three research laboratories, located in three different places (two in UK and one in US); in spite of the fact that the HSE activities require great collaboration among all researchers. For improving integration, they have connected the three 
laboratories through a distributed personnel database, accessible by every researcher. The database memorises skills, specialisation, know-how and previous project experiences of all employees of the three laboratories, so that everybody in the department can access the information about researchers as if they were working in a unique small group. For example, it is possible to identify who was previously involved in a specified problem, who has the required expertise, etc.

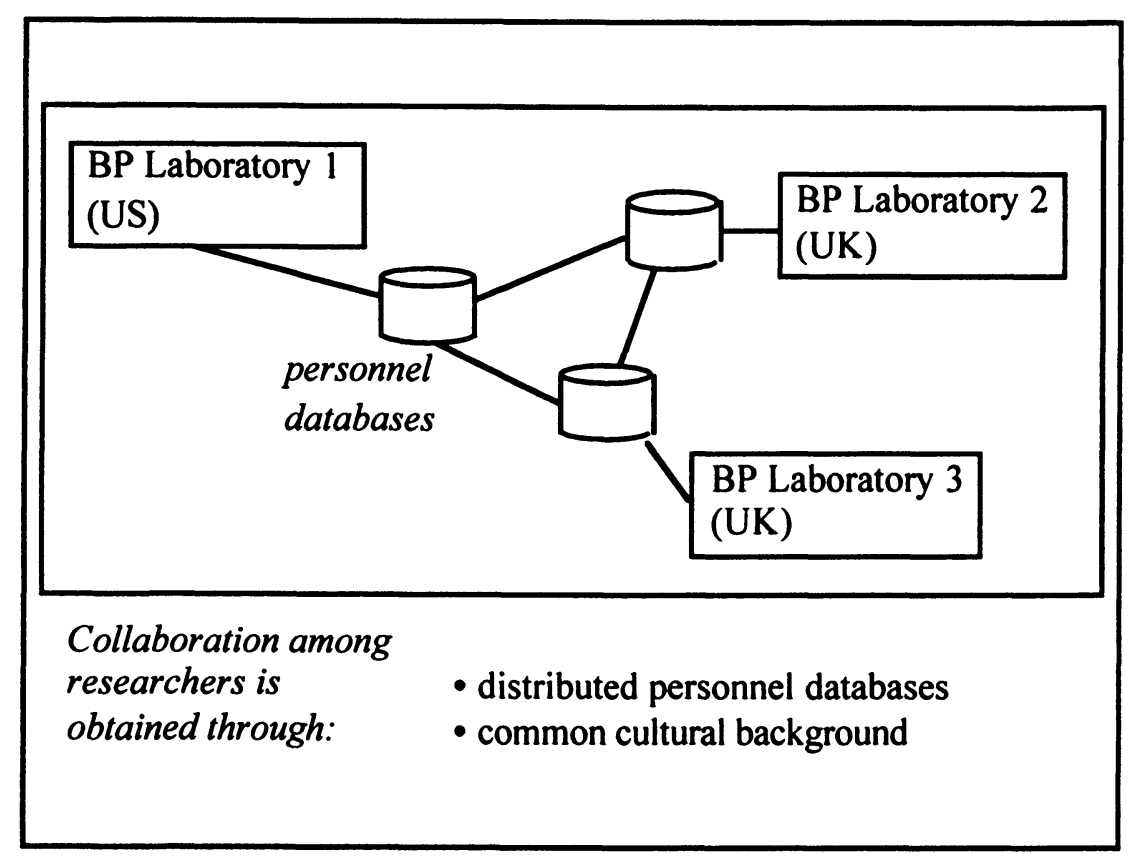

Figure 2 The ODSS of British Petroleum

\subsection{IT as enablers of organizational relations}

The previous example has shown the capability of IT to foster the creation of organizational relations. According to the proposed relational method for organizational analysis, Information Technology can be considered as a tool; just like many others mechanisms. Nevertheless, due to its great possibilities, IT is a special tool, able to foster organizational relations. New IT tools could be enablers of modern organizational mechanisms, based on flexible and effective relations. In some cases the network of organizational relations would not be possible without IT based tools.

An Italian example is provided by the network organization of Benetton, outline in figure 3. This company is composed of a central node, situated in Treviso, about 500 small factories, and a lot of shops all over the world. The small factories and the shops are linked to the central node by different kinds of formal contracts (most 
of them are franchising contracts) and by a computer network. The computer network supports the collection of orders from the shops to the head office, so as to make the production planning easy. Through the information network, the central node informs the linked factories about the quantity and the quality of goods to be produced. Without IT tools, the network of organizational relation in Benetton would perhaps be very different: it would involve a limited number of nodes and the exchange of information would not be as easy as now.

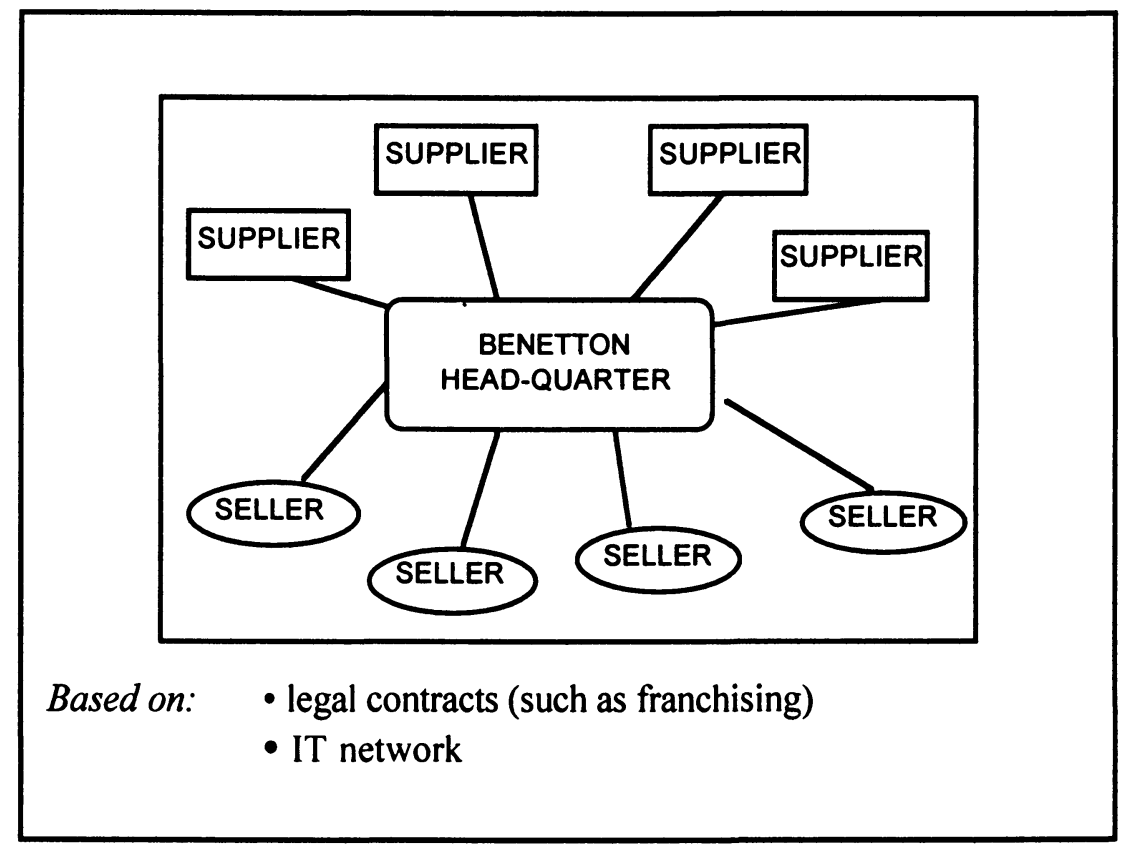

Figure 3 The Benetton network organization

But the design of IT tools is not enough for the implementation of effective organizational relations. The proposed method of analysis of organizational relation prescribes a coupled design of the four axes: tools, goals, norms, cultural background. In particular the design of Information Technology for supporting existing organizational relations may fail when the technical design of the tools is not joined to the analysis of the remaining three axes of the relation. The relational method provides two main design principles for preventing IT failures:

\section{Coherence among tools}

Effective relations use several tools at the same time. This means that IT design should look for the coherence of IT tools with the other ones. For example one of the most natural tools in group decision activities is interpersonal contact: the design and introduction of Group Decision Support Systems (IT tools) has to look 
for integration with the existing interpersonal contact tool, for example by planning periodic face-to-face meetings (Vogel and Nunamaker, 1990). The main causes of failure of most GDSS are that they do not respect the required coherence: often groups using GDSS are "co-located yet remote" (Whitaker, 1994); participants in electronic meetings "report a feeling of dissociation from others, due to the lack of interaction" (Bannon, 1997).

IT tools (as well as the other tools) need to be coherent with the remaining axis of goals, norms, cultural background.

For example, the organizational relations of franchising (among a central company and several dispersed shops) generally have the following characteristics: formal and easy to measure goals, standard norms (all the norms are clearly defined in the franchising contracts), limited cultural background (the two actors must share a limited knowledge about the product and about some marketing techniques). This franchising organizational relation may be supported through a computer network, that enables the exchange of a limited set of structured data (for example data about sales, catalogues, etc.). On the contrary the organizational relation among two remote R\&D units is based on: non rigidly defined goals, formal and informal norms (they ca not negotiate a rigid contract), a vast cultural background (that continuously changes with new information/discoveries). IT support must enable the non structured exchange of information, data and hypertext documents(E-mail is a proper form of support).

\subsection{Two dilemmas for organizational relation design: formal/informal and community/society.}

Each of the four components of a relation proposed with the AGIL adapted model can be described along two axes: formalinformal and community/society. This classification allows a better description of the four elements of the relation and the analysis of the role of Information Technology in defining the characteristics of relations in network organizations.

The axis formalinformal underlines that in every relation there are both formal and informal elements (Donati, 1991). A hierarchic relation uses for example both formal tools (such as rewards, reproof, punishment, etc.) and informal tools (authority, influence, etc.); it follows both formal goals (formal objectives, coordination and control of subordinates, etc.) and informal ones (encouraging, stimulating, etc.). Every relation has aspects of both types, but in some cases there are more formal elements in the relation, in some cases more informal ones.

The binomial community/society was introduced by F. Tonnies (1887) in the social field. The word community (Gemeinschaft) indicates an aggregation based on traditional behaviour and on the common will of people. In a simple way, the community is the most basic and primary form of association of people. Key words for understanding the meaning of community are friendship, village, solidarity, etc. 
Society (Gesellschaft) is based on common accords, on legal rules and on contracts. It is a more complex form of aggregation and requires a formal agreement on the way it takes place.

Organizations are aggregations of societal type: they are based on accord and contract among individuals that follow their interests in the rational way. Nevertheless, in an organization there are also some aspects of community type, since organizations answer some basic human needs (the need for communication, the need for association, etc.).

Table 2 Examples of social norms and legal rules are born in community and in society (Donati, 1991)

\begin{tabular}{l|ll}
\hline & Community & Society \\
\hline Social order & Consensus & Contract \\
Law & $\begin{array}{l}\text { Custom } \\
\text { (Tradition) }\end{array}$ & State, Government \\
\hline
\end{tabular}

These two criteria (formal/informal, society/community) are the basis for the definition of different types of relations as exemplified in table 3.

Table 3 Example of intersection between formal/informal and community/society.

\begin{tabular}{l|ll}
\hline & Formal & Informal \\
\hline Community & $\begin{array}{l}\text { Relation between a trade } \\
\text { union officer and a worker }\end{array}$ & Relation between two friends \\
Society & $\begin{array}{l}\text { Relation between a } \\
\text { supervisor and a subordinate } \\
\text { in an assembly line }\end{array}$ & $\begin{array}{l}\text { Relation among the members of } \\
\text { a surgical team. }\end{array}$ \\
\hline
\end{tabular}

The two proposed oppositions are particularly relevant for the analysis of the role of IT in the innovative network organizational model, which represent one of the main goals of this paper. Information technology instruments are, in fact, formal and, at the same time, societal tools: IT pushes a relation from formal to informal and from community to society. IT tools are based on standards of communication (protocols) that specify the form of messages to exchange. IT-based 
connections have to respect the limits of electronic media; communication regards only keyboard (or mouse) imputable data and screen suitable information. Through IT tools, interaction become more efficient and more oriented to organizational objectives (society purpose) than "face-to-face relations". Communication become colder and less rich: only formal messages are exchanged among nodes.

Groups using electronic brainstorming tools are, for example, more productive than the ones using traditional brainstorming and with electronic brainstorming tools groups generate more good ideas, that are the formal and society purposes of those teams. At the same time, in brainstorming supported by electronic tools, there is not more oral communication: participants can talk if they want but they choose not because they are concentrating on their screen. This is a disadvantage because it reduces the opportunity for personal interaction while the richness of oral communications gets lost (i.e. the informal and community aspects are lost) (Gallupe and Cooper, 1993). The introduction of electronic brainstorming is good for the efficiency of the group (number of ideas produced), but it is not good for the ability of the group to work outside the computer structured logic schema, i.e. the team is less flexible.

Roughly speaking, IT causes change from a man-to-man relation to a triple connection: man-to-machine, machine-to-machine, machine-to-man. For this reason, it has been seen that in groups using GDSS periodic face to face meetings are required to improve the quality of decisions and the efficiency of communications (Vogel and Nunamaker, 1990).

\section{APPLICATIONS OF THE PROPOSED RELATIONAL MODEL AND} A CASE STUDY.

Empirical research have been conducted in order to reveal the presence and the effectiveness of network organization in a sample of manufacturing companies. The analysed organizations have been decomposed in nodes (organizational units with similar objectives and belongings to the same organizational process) and the links among nodes have been discovered for analysing existence and effectiveness of network organizations. Relational matrices were used in order to summarise the networking propulsion of each company. Empirical research revealed that the presence of network organization was positively correlated with effectiveness and flexibility. Moreover the analysis discovered an extension of propulsion toward network organizational forms in last few years. More detailed case studies have been conducted in two organizations: in the Italian company Olivetti and in an Italian Health Service. These case studies have tested the proposed relational model for organizational analysis and have proved its validity. 


\subsection{The DSS design for an Italian health agency}

In this section we will describe the application of the proposed relational method for implementing a managerial DSS in an Italian public health agency. The case regards the psychological and social division of the health agency. This division provides a town of about 120,000 persons with psychological services and social work.

An expert psychologist is the manager of the division. His task is very broad and regards the technical supervision of other employees, the institutional deputation of the division, the psychological consulting, the planning and control of the division activities. This psychologist is one of the four top managers of the health agency: so he is always very busy with his work. The other psychologists and social employees of the division are organized in nine teams. Three teams deal with child patients (from 0 to 14 years old), three with adolescent patients (from 14 to 18 years old) and three with senior patients (more than 18 years old). Each group of three teams has a sector-head psychologist and each team has a team-head psychologist. Moreover, the division is composed of some administrative employees, working on a central office under the direction of an administrative boss.

At the beginning of 1994, the division manager asked a consulting-team (composed of the authors of this paper) to design and to implement a managerial DSS. The introduction of a managerial DSS in this psychological and social division required a complex process of organizational change and political mediation in the organization. The initial decisional user requirements were modified and completed with other features in order to make the organization accept the new system.

\section{The analysis of decisional and information needs}

The division manager asked for a managerial DSS to support his planning activities. He wanted an IT based tool providing help for decision making to create the annual plan as well as the three-year activity planning. The manager needed an accurate and synthetic description of the past activities of his division (data such as: the number of patient with certain diseases, the frequency of certain psychological treatment, the correlation between external conditions and certain diseases, etc.) in order to formulate a good organizational plan for the future.

A DSS was designed, based on data collected about the team's activities. At least once a week, each psychologist and social employee would have provided data about his own patients (diagnosis, therapy, results, etc.), through structured interfaces for data entry. Through the tool, the manager would have extracted the required statistical information from data collected.

Uncertainty about which data to collect was one of the main problems in this design phase. The information required by the manager may vary in relation to new planning needs. This necessitates designing a flexible system. Thus the 
possibility for handling new user-defined queries was introduced and the data base was built in order to be easy to modify.

When the news of the introduction of the new DSS circulated in the organizations, psychologists and social employees expressed their strong opposition against the new IT system. Some causes of opposition were easy to foresee while others were less clear. In order to prevent system failure, the division was analysed according to the users' perspective on their job changes, and according to the Relational Perspective.

The analysis of job modification.

The DSS introduction would have changed the job and the work habits of the psychological and social employees. First, most of the psychologists and social employees had no expertise in computer usage. Even if the data entry interfaces were user friendly and easy to use, the DSS would have required some minimum computer learning. In most employees the need for a training process caused an "a priori" refusal of the system: some employees refused to have to learn a new subject. Second, the DSS was designed for managerial planning activities. Psychologists and social employees would have obtained no direct benefits from the system, whereas they would have taken on the data entry activity. Even if interfaces were designed in order to minimise the time required for data entry, psychologists and social employees had to modify their work organization for maintaining the data base. This caused a diffused refusal of the new DSS.

\section{The analysis of the network of organizational relatior al.}

The analysis of the network of relations in the division re zaled a third cause of opposition against the DSS. Four nodes were detected with the criterion of relational homogeneity:

- the manager.

- the administrative employees

- the team head psychologists and some few other social employees

- the remaining psychologists and social employees.

The discovered relations among the four nodes are complex and composed of several dimensions. In the following, we provide only a synthetic description for case understanding (Figure 4 and Table 4, below). The relational method proposed in section 4 is used.

Relations among node 2 and nodes 1, 3, 4.

The node 2 is linked with a similar relation to all the remaining nodes.

Tools: First of all, the relation is based on the work flow of documents from/to administrative office to/from psychological teams (technical dimension). Second, there is an important social link. Psychologists appreciate administrative 
employees, because they relieve psychologists from all the fastidious bureaucratic problems and duties. Administrative employees hold psychologists and social employees in esteem, because of their professional preparation and their ability to deal with patients.

Goals: The organizational goal of this relation is to provide psychology and social employees with administrative and bureaucratic support.

Rules: The flow of document has to respect some formal rules, decided by the health-agency top-management as well as by external public agency (for example national or regional government). The social esteem link has established also unwritten rules of mutual help.

Cultural background: Employees belonging to node 2 share with the rest of the employees the same organizational culture: assumptions about top management, stories and legend regarding the public health, etc. As a result, the emerging organizational relation is characterised by a mutual liking that enables a good work collaboration.

\section{Relations between node 1 and node 3 .}

Tools: The relation between node 1 and node 3 is based on three main tools: (a) hierarchical link: node 1 is the supervisor of the components of node 3; (b) periodic meetings. The division manager (node 1) gives psychological consulting to team head psychologists (node 3): face-to-face meetings take place at least once a week. The number of these meetings is considered insufficient by the team's head psychologists: in fact, the division manager is very busy also with his institutional task (he is one of the top managers of the health agency). (c) psychological link of esteem: the psychologists and social employees of node 3 recognise the professional capability of the division manager and his psychological expertise.

Goals: Two main organizational goals are assigned to this relation: (a) the operational control of psychological teams; (b) the psychological consulting to team-head psychologists. This relation between the division manager and the teamhead psychologists should ensure the quality of the psychological cares.

Rules: There are a number of sources of rules: (a) formal hierarchy rules, (b) authority rules, deriving from the professional capabilities of the division manager, (c) professional rules, due to general practice and theoretical models in psychological consultancy.

Cultural background: The division manager and the team head psychologists share the same organizational culture (such as in the relation between node 2 and nodes $1,3,4)$ and a common psychological background. The resulting relation may be defined "neutral". There is coherence among the four dimensions of tools, goals, rules and cultural background that enables an effective work collaboration. Nevertheless, the limited number of meetings causes frequent small conflicts, so that the relations remain quite cold. 


\section{Relations between node 1 and node 4.}

Tools: The relation between node 1 and node 4 is based on the hierarchical authority of the manager.

Goals: The organizational goals of this relation are the same as in the relation between node 1 and node 2 .

Rules: The formal hierarchical norms regulate the relation.

Cultural background: The two nodes share a common organizational background. Moreover some of the employees belonging to node 4 are psychologists and they share a common psychological culture with the division manager.

Table 4 The analysis of the network of relations through the proposed relational method

\begin{tabular}{|c|c|c|c|c|}
\hline & $1<=>3$ & $1<=>4$ & $3<=>4$ & $2<=>1,3,4$ \\
\hline Tools & $\begin{array}{l}\text { - Hierarchy link } \\
\text {-Psychological } \\
\text { consulting } \\
\text { meeting }\end{array}$ & - Hierarchy link & $\begin{array}{l}\text { - Hierarchy link } \\
\text { - Cooperation } \\
\text { for patients } \\
\text { caring } \\
\text { - "Clan" links }\end{array}$ & $\begin{array}{l}\text { - Flow of } \\
\text { documents } \\
\text { - Interpersonal } \\
\text { links }\end{array}$ \\
\hline Goals & $\begin{array}{l}\text { - Control and } \\
\text { support of } \\
\text { psychological } \\
\text { teams }\end{array}$ & $\begin{array}{l}\text { - Control and } \\
\text { support of } \\
\text { psychological } \\
\text { teams }\end{array}$ & $\begin{array}{l}\text { - Support of } \\
\text { psychologists } \\
\text { and social } \\
\text { employees }\end{array}$ & $\begin{array}{l}\text { - Administrative } \\
\text { support }\end{array}$ \\
\hline Rules & $\begin{array}{l}\text { - Hierarchical } \\
\text { rules } \\
\text { - Psychological } \\
\text { competence of } \\
\text { the Division } \\
\text { Manager } \\
\text { - Professional } \\
\text { rules on } \\
\text { psychological } \\
\text { consulting }\end{array}$ & $\begin{array}{l}\text { - Hierarchical } \\
\text { rules }\end{array}$ & $\begin{array}{l}\text { - Hierarchical } \\
\text { rules } \\
\text { - Professional } \\
\text { rules regarding } \\
\text { psychological } \\
\text { methodologies } \\
\text { - Mutual help } \\
\text { rules }\end{array}$ & $\begin{array}{l}\text { - Hierarchical } \\
\text { and bureaucratic } \\
\text { rules } \\
\text { - Mutual help } \\
\text { rules }\end{array}$ \\
\hline $\begin{array}{l}\text { Cult. } \\
\text { Bkg. }\end{array}$ & $\begin{array}{l}\text { - Organizational } \\
\text { culture } \\
\text { - Psychological } \\
\text { background }\end{array}$ & $\begin{array}{l}\text { - Organizational } \\
\text { culture } \\
\text { - Psychological } \\
\text { background }\end{array}$ & $\begin{array}{l}\text { - Organizational } \\
\text { culture } \\
\text { - Psychological- } \\
\text { team culture }\end{array}$ & $\begin{array}{l}\text { - Organizational } \\
\text { culture }\end{array}$ \\
\hline
\end{tabular}

The analysis reveals a low coherence among the four dimensions: in particular the important organizational goals are not coherent with limited hierarchy based tool. This relation should be similar to the relation between node 1 and node 3 , but because of work load problems, the division manager gives direct psychological consulting only to the team head psychologists. The remaining psychologists and social employees (node 4) have few opportunities for working together with him. 
This causes a feel of mistrust of node 4 toward node 1 . In the resulting relation, work collaboration is very difficult.

\section{Relations between node 3 and node 4.}

Tools: The link between node 3 and node 4 is based on: a) hierarchical supervision of members of node 3 toward components of node 4; (b) technical interdependencies. Between the activities of the two nodes there are complex interdependence as they work together in the teams for patients caring; (c) social links: the team based organization and the particular task (the care of social and psychological disease) has developed a strong cohesion among team members. These teams may be correctly defined as "clans" (Ouchi, 1980).

Goals: Node 3 has the organizational goal to control and to support the node 4, in order to ensure the quality of the psychological team activities.

Rules: Formal hierarchical rules exist but they are scarcely used. The teamworking has developed unwritten rules of trust, commitment to mutual help, friendship. Moreover psychological methodologies provide a framework for regulating the teamwork.

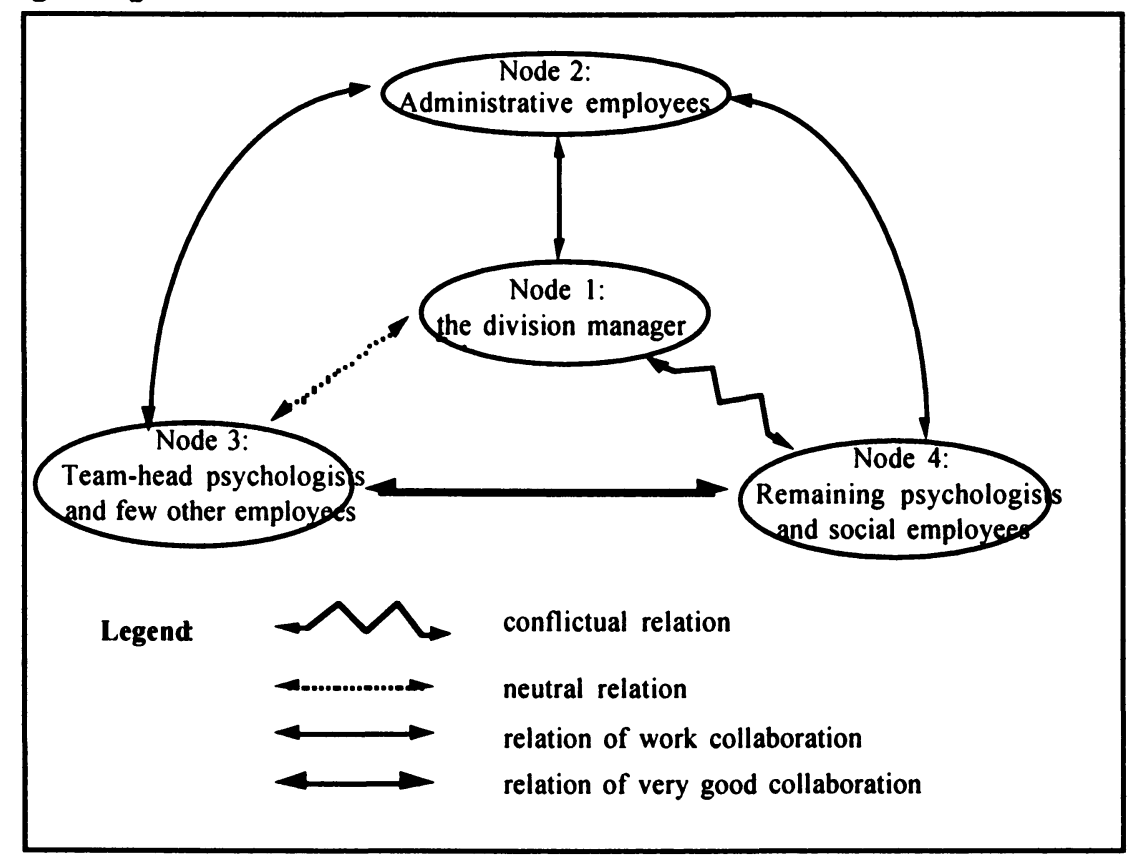

Figure $4 \mathrm{~A}$ synthesis of the network of relations in the social and psychological division.

Cultural background: The two nodes share the common organizational culture. 
They are also unified by the team specific culture: for example stories, past "epic" episodes and "legend" regarding the team circulate among team members. Moreover the daily working together has developed a specific slang.

The resulting relation is very warm and it enables a strong work collaboration.

\section{Conclusions of the network of relations analysis in the Health Agency}

The managerial DSS was perceived as a relational instrument. Existing relations influenced the "political" judgement to its introduction in the organization. Psychologists and social employees of node 4 were particularly against the DSS. Because of the existing relation between node 4 and node 1 (Figure 5), they saw the new IT tool as an instrument of control of their activity imposed by the manager.

The opposition of other psychologists and employees (belonging to node 3 ) was less strong, and limited to work habits change reasons. Nevertheless the warm relation linking node 3 to node 4 prevent psychologists belonging to node 3 from taking sides in favour of the DSS.

\subsection{A case study of a big Italian company: Olivetti DCI}

In the following section, we will describe a simple application of the relational approach to network organizations. The aim of this description is to show how a relational approach can explain the complex aspects involved in an internal network organization. For this purpose we describe the case study of a big Italian company.

As we have seen, internal network organizations are among the new trends in organization form. Organizational unity of internal network organizations are partially autonomous and can connect to one another in some way. In the Italian Commercial Department of Olivetti (Olivetti DCI), every organizational unit has a budget of its own, that it can increase by selling its performances to other organizational units or decrease by buying services from other nodes. In this way, an internal market develops among organizational units. This quasi-market joins to the existing hierarchical relations.

A network model can describe the contemporary presence of both hierarchy and market. We can model organizational units as nodes connected by two kinds of relations: hierarchical relations and market ones. The formal tools of hierarchical relations are the usual instruments of an authority/dependency connection, as punishment and rewards; their main goals are control and co-ordination of employees. The formal tools of market relations are the contracts of internal exchanges; their goals are sales and/or purchases of services, so as to increase the organizational unit's budget. But, in fact, connections among organizational units are more complex than market or hierarchy relations: those relations still exist also when market transactions are finished and they create a valid system of collaboration among the different employees of the firm.

Comprehension of these phenomena requires the knowledge of the organizational culture of Olivetti DCI, which presents the following characteristics: 
it is oriented to collaboration and informal contacts; individual competition is low; the considered and examined relations use also informal tools and follow community purposes. Thus, beside the introduction of internal market, a complex network of organizational and interpersonal relations exists.

Roughly, we can propose the following developing cycle in this case study, explaining the two elements for this partial network organizational model (internal market and interpersonal relations):

- At the beginning relation is built by two organizational units, with the objective of sales (or purchases) of services (society goal). Instruments are internal contracts (formal tools).

- After the two organizational units have drawn up the contract, interpersonal contacts emerge and become the main tool of the relation (people belonging to the two different organizational units work together).

- Social values and organizational culture change the goals of relations: collaboration among individuals becomes informal and of a community-type: the two organizational units collaborate not only for market exchange but also for every kind of problem solving.

- When contractual objectives have been achieved, formal tools stop working but informal ones remain still alive. A community-type relation connects the two organizational units at least for some months (sometimes persistently).

A description of the various different relations revealed and discovered in the simple case study at Olivetti DCI, is summarised in Table 5, along the parameter society/community.

Table 5 The characteristics of relations in Olivetti DCI

\begin{tabular}{l|ll}
\hline & Society & Community \\
\hline Tools & - market contracts & -interpersonal contacts \\
Goals & $\begin{array}{l}\text { efficiently sale and/or } \\
\text { purchase of services }\end{array}$ & - problem solving \\
Organizational rules and & $\begin{array}{l}\text { rules and norms of } \\
\text { culture }\end{array}$ & - collaboration \\
behaviour & - informality \\
\hline
\end{tabular}

As a result of this process, every member of Olivetti DCI is a node of a complex network: he or she can use relations with other nodes for problem solving, for finding information and contacting experts. Some employees are appreciated fundamentally either because they are able to connect a great number of nodes or because of their capacity to use the best relation for solving the worst problems. The set of relations they can establish changes and enlarges their role in the network.

The use of the proposed relational analysis has made clear the complex dynamics of Olivetti DCI internal network. From the given description indications 
for the management can be obtained, both for operative aspects and for IT design: as regards operative aspects, relational analysis for example finds out the key nodes (i.e. people or organizational units connected to the large number of nodes): those nodes for example have an important role in the most important projects because of their connection capability.

As concerns IT design, the analysis shows for example that the introduction of an information network in Olivetti DCI could sort positive effects as regards the interchange of information among organizational units, but it may not (and it must not) eliminate the described interpersonal contacts that give an important support to the capacity of problem solving and decision making in Olivetti DCI.

\section{CONCLUSION}

Internal network organizations are among the most innovative forms of organizations; IT and especially DSS and GDSS play an important role in their design and development A network is made up of two components: nodes and links. The new competitive features of the present economic environment require enlargement the autonomy of the nodes. The enlarged autonomy requires the improvement of the skills and the know-how of every node and it needs to extend the amount of available information on environmental variables and opportunities. One of the ways to obtain the capacity and the know-how required by every node is to establish links with other nodes: a link allows the exchange of information, services, know-how, etc., between two (or more) nodes, through organizational relations, information channels, interpersonal contacts.

This paper has proposed a method for the design of a network organization based on the concept of relation. A relation is made by two nodes connected by a link. The concept of relation regards both the characteristics of the two nodes and the properties of the link itself. The basic elements of a relation are the tools that support the relation, the goal of the nodes, the organizational rules of the organization group and the organizational culture. Each of these four elements can be described along two axes: the opposition formal/informal and the opposition community/society.

In the paper, the role of IT in the network organizations has been analysed according to three aspects. First of all, IT (especially DSS and GDSS) improves the autonomy of every node: it allows the sharing of information and experience among organizational units, it provides a common set of problem solving methodologies and increases the knowledge of the node. The Digital Delta methodology for example allows the sharing of experience among the organizational units, and so it improves the know-how and the capacity of every node. Secondly, IT provides tools for creating links and relations. Computer networks permit the exchange of data among remote points. GDSSs provide a lot of facilities to improve communications and to establish relations, for example with remote conferences, decision rooms, electronic brainstorming instruments. 
The network of Benetton is an example of how IT can support the relations in network organizations. Thirdly, the impact of the use of IT is to push a relation from informal to formal and from community to society. The paper shows that this improves the efficiency of the relation, but reduces the richness of the interaction: the design of IT tools has to solve the trade-off between those two aspects. The choice of the degree of the introduction of IT depends on the importance of existing informal and community aspects and/or on the lack of communication links.

\section{REFERENCES}

Applegate, L. M., Cash J. I. Jr. and Mills D. Q. (1988) Information Technology and Tomorrow's Manager, Harvard Business Review.

Bannon, L. (1997) CSCW - A challenge to certain (G)DSS perspectives on the role of decisions, information and technology in organisations. This volume.

Ciborra, C. (1993) Teams, Markets and Systems. Cambridge University Press, Cambridge.

Ciborra, C., Migliarese, P. and Romano P. (1984) A methodological inquiry of Organizational Noise in Socio-technical Systems, Human Relations, 37, 56588.

Donati, P. (1991) Teoria relazionale della società, Franco Angeli, Milano.

Drucker, P. F., (1988), The Coming of New Organizations, Harvard Business Review, January-February.

Ferioli, C. and Migliarese P. (1996a) A DSS Design method based on organizational change, in Implementing Systems for Supporting Management Decisions (eds P. Humphreys, L. Bannon, A. McCosh, P. Migliarese and J. C. Pommerol). Chapman and Hall, London.

Ferioli, C. and Migliarese, P. (1996b) Supporting organizational relations through information technology in innovative organizational forms. European Joumal of Information Systems 3 No. 5.

Ferioli, C. and Migliarese P. (1994) The role of IT and GDSS in internal network organizations. in Proceedings of IFIP WG 8.3 Working Conference on Decision Support in Organisational Transformation (eds. B. Mayon-White, S. Ayestaràn and P. Humphreys) San Sebastian.

Gallupe, R. B. and Cooper W. H. (1993) Brainstorming Electronically, Sloan Management Review.

George J. F. and Nunamaker, J. F. (1992) ODSS Information Technology for Organizational Change, Decision Support Systems, 8

Humphreys , P., Bannon, L., McCosh, A., Migliarese, P. and Pommerol, J. C. (1996) Implementing Systems for Supporting Management Decisions Chapman and Hall, London..

Lee, R. M., McCosh A.M. and Migliarese P. (1988) Organizational Decision Support Systems, North Holland, Amsterdam. 
Miles, R. E. and Snow, C. C. (1986) Organizations: New Concepts for New Forms, California Management Review, 3, Spring.

Migliarese, P. (1984) Knowledge representation and organizational noise: an organizational framework about Decision Support Systems, in Knowledge representation for decision support systems (ed. J. Hawgood), North Holland, Amsterdam..

Migliarese, P. (1992) Sistemi di supporto per i processi decisionali, in Progettare e gestire l'impresa innovativa (eds. R. Filippini, G. Pagliarani and G. Petroni), ETAS Libri, Milano.

Migliarese, P., et al. (1995) Improved communications and collaborations among tasks induced by Groupware. Decision Support System Journal, 14, 237-250.

Parsons, T., Smelser N. J., (1956), Economy and Society, Free Press, New York.

Tonnies, F, (1887), Gemeinschaft und Gesellschaft, Leipzig.

Vogel, D., Nunamaker, J. F., Jr, George, J. F. and Dennis, A. R. (1988), Group Decision Support Systems, in Organizational Decision Support Systems (eds. R. M. Lee, A.M. McCosh and P. Migliarese) North-Holland, Amsterdam.

Vogel, D. and Nunamaker, J. F. (1990) Group Decision Support Systems impact: multi-methodological exploration, Information and Management, 18.

Zigurs, I. (1989) Interaction Analysis in GDSS Research: Description of an Experience and Some Recommendations, Decision Support Systems, 5.

\section{BIOGRAPHIES}

Piero Migliarese graduated in electrical engineering at Politecnico of Milano. He is full Professor of Managerial Economics and Organizational Systems and teaches at Politecnico of Milano, Italy and at University of Calabria, Italy. He is a founding member of IFIP 8.3 Working Group on Decision Support Systems. His research interests are regarding innovative organizational models, information systems, group decision support systems and work support systems. At present he is proposing conceptual models regarding co-ordination and co-operation support coming from information technologies. He has published various papers on these subjects in national and international journals. He has edited two books on the organizational perspective of Decision Support Systems, published by North Holland and Chapman and Hall.

Claudio Ferioli graduated in Electrical Engineering at Politecnico of Milano and worked in the field of Information Technology as a CASE expert. Since 1993 he has been working in the research activities of the Department of Economics and Production, Politecnico of Milano. He participates in a number of research projects about the emergence of new organizational models and the role played by information technologies in implementing organizational changes. He collaborates with public health organizations for management consultancy and software implementation. He is a member of the IFIP 8.3 Working Group on Decision Support Systems. 